\title{
Research on the fixed elasticity flying of high speed aircraft Guangbin $\mathrm{Wu}^{\mathrm{a}}$, Lingling Wang and Huali Wu
}

Department of control engineering, Naval aeronautical and astronautical University Yanti, 264001

awuguangbin1024@126.com

Keywords: aircraft; stability; control; fixed elasticity flying; numerical simulation

\begin{abstract}
The elasticity is a very special factor considered in modeling of supersonic aircraft, and it is usually neglected in supersonic aircraft. Since it will cause the force and moment of the aircraft totally different, so it increased the complexity and difficulty of hypersonic aircraft. To simplify the analysis and to testify the rightness of the air coefficients of aircraft model, a kind of fixed elasticity virtual flying condition is proposed and the numerical simulation is done with computer program according to the proposed model and coefficients. Finally, the simulation result is shown to analyze the dynamic performance and characteristic of the proposed method.
\end{abstract}

\section{Introduction}

The changing of elastic form is the typical difference between the hypersonic aircraft and the non-hypersonic aircraft ${ }^{[1-5]}$. Elastic deformation doesn't need to be considered in modeling the traditional hypersonic aircraft. Elastic deformation is the reason that the hypersonic aircraft cannot be controlled, because the large coupling between the changing of elastic form and attack angle, and the elastic form itself also has impact on the stress of aircraft, so the attack angle changes. We don't need to consider the elastic modality on the research of verifying the model of hypersonic aircraft, according to the principle which is from easy to complex ${ }^{[6-10]}$. That is the flying test on the fixed elastic modality as well. The simulation results indicate that the changing characteristics of the posture and attack angle, which caused by hypersonic aircraft itself, is the real dynamic character. This character is more complex than low speed aircraft. The results indicate the conclusions right above. That's can say the complicated of hypersonic aircraft caused not only by the elastic form but also the combination effect of engine, hypersonic and elastic deformation.

\section{Model Description}

Considering the elastic shape structure, a kind of pitch channel hypersonic aircraft model built according to Lagrange equation is released by USA air force as followed:

$$
\begin{gathered}
\dot{V}=\frac{T \cos \alpha-D}{m}-g \sin \gamma \\
\dot{\phi}=-2 \varsigma \omega_{n} \phi-\omega_{n}^{2} \phi+\omega_{n}^{2} \phi_{c} \\
\dot{\gamma}=\frac{L+T \sin \alpha}{m V}-\frac{g \cos \gamma}{V} \\
\dot{\alpha}=q-\dot{\gamma} \\
\dot{q}=\frac{M}{I} \\
\dot{h}=V \sin \gamma \\
\ddot{\eta}_{i}=-2 \varepsilon_{m} \omega_{m i} \dot{\eta}_{i}-\omega_{m i}^{2} \eta_{i}+N_{i}
\end{gathered}
$$

Where 


$$
\begin{gathered}
T=\bar{q} s\left(C_{T \phi} \phi+C_{T}+C_{T}^{\eta} \eta\right), D=\bar{q} S C_{D}, \\
N_{i}^{\eta}=\left[N_{i}^{\eta_{1}}, 0, N_{i}^{\eta_{2}}, 0, N_{i}^{\eta_{3}}, 0\right] \\
C_{j}^{\eta}=\left[C_{j}^{\eta_{1}}, 0, C_{j}^{\eta_{2}}, 0, C_{j}^{\eta_{3}}, 0\right], j=T, M, L, D \\
L=\bar{q} S C_{L},, \\
M=z_{T} T+\bar{q} S \bar{c} C_{M}, \\
N_{i}=\bar{q} S C_{N_{i}} \\
C_{N_{i}}=C_{N_{i}}^{\alpha^{2}} \alpha^{2}+C_{N_{i}}^{\alpha} \alpha+C_{N_{i}}^{\delta_{e}} \delta_{e}+C_{N_{i}}^{\delta_{c}} \delta_{c}+C_{N_{i}}^{0}+N_{i}^{\eta} \eta \\
=1.4013 * 10^{3} \alpha^{2}+4.5337 * 10^{3} \alpha+198.6 \delta_{e}+230.48 \delta_{c}+117.52 \\
+2.734 * 10^{3} \eta_{1}-1.18 * 10^{3} \eta_{2}+110 \eta_{3} \\
C_{N_{2}}=C_{N_{2}}^{\alpha^{2}} \alpha^{2}+C_{N_{2}}^{\alpha} \alpha+C_{N_{2}}^{\alpha_{e}} \delta_{e}+C_{N_{2}}^{\delta_{c}} \delta_{c}+C_{N_{2}}^{0}+C_{2}^{\eta} \eta \\
=-5.0227 * 10^{3} \alpha^{2}+2.8633 * 10^{3} \alpha+1.2465 * 10^{3} \delta_{e}+0 \delta_{c}-44.021 \\
+0.98 * 10^{3} \eta_{1}+2.89 * 10^{3} \eta_{2}+58 \eta_{3} \\
C_{N_{3}}=C_{N_{3}}^{\alpha^{2}} \alpha^{2}+C_{N_{3}}^{\alpha} \alpha+C_{N_{3}}^{\delta_{e}} \delta_{e}+C_{N_{3}}^{\delta_{c}} \delta_{c}+C_{N_{3}}^{0}+N_{3}^{\eta} \eta \\
=2.3048 * 10^{3} \alpha^{2}+3.2072 * 10^{3} \alpha+0 * 10^{3} \delta_{e}+0.9763 * 10^{3} \delta_{c}+68.73 \\
+0.258 * 10^{3} \eta_{1}+0.91 * 10^{3} \eta_{2}+7560 \eta_{3}
\end{gathered}
$$

And $V$ is speed, $\gamma$ is the speed angle, $\alpha$ is attack angle, $Q$ is the attitude angle speed, $h$ is the height. $\phi$ is the oil supplying factor, $\delta_{c}$ is the duck wing and $\delta_{e}$ is the lift rudder.

\section{Simulation settings of free flying}

In order to testify the rightness of the model of hypersonic aircraft, choose a initial height as $h_{0}=85000 * 0.3048$, initial speed as $V_{0}=7846 * 0.3048$, initial attack angle as $\alpha_{0}=0.0174$, and other initial state as: $\gamma_{0}=0, q_{0}=0, \eta_{10}=0.4588 * 0.3048 * 14.59, \eta_{20}=-0.08726 * 0.3048 * 14.59$, $\eta_{30}=-0.03671 * 0.3048 * 14.59$, and set the oil supplying law as constant as $\phi=\phi_{c}=0.1$, and choose the control as $\delta_{c}=\delta_{e}=0$, then the fixed elasticity flying test can be done by numerical simulation and simulation time is set as 0.5 second.

\section{Numerical Simulation and result analysis}

With above model and air coefficients and set initial condition as above paragraph and write a program with $\mathrm{m}$ language in Matlab software, then the simulation can be done and simulation results can be shown as following figures. 


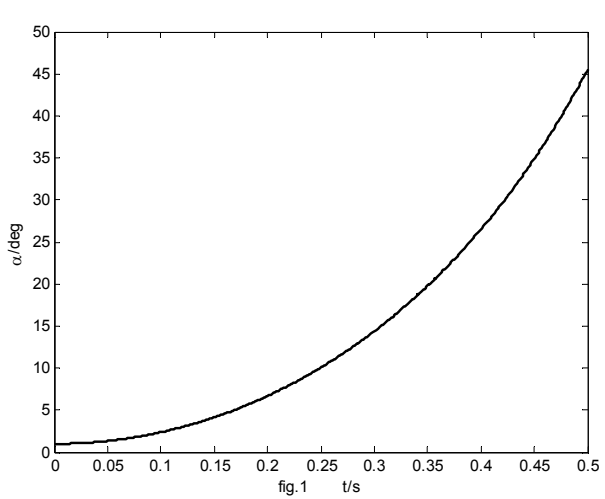

Fig 1 The curve of attack angle

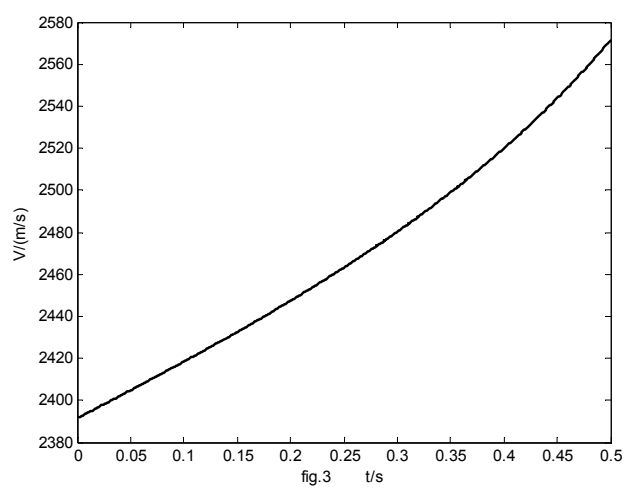

Fig 3 The curve of speed

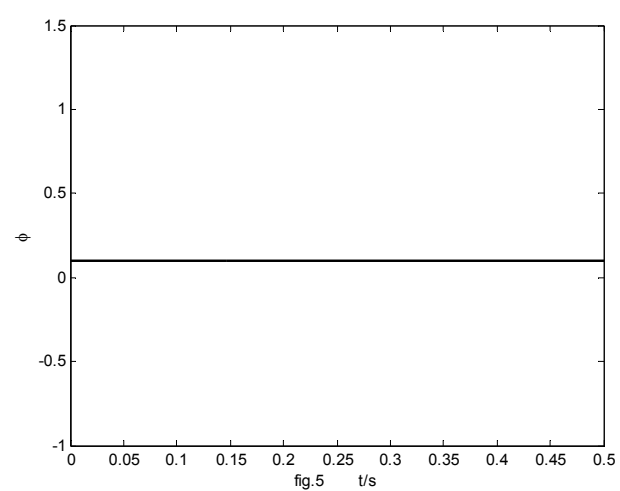

Fig 5 The curve of oil supplying factor

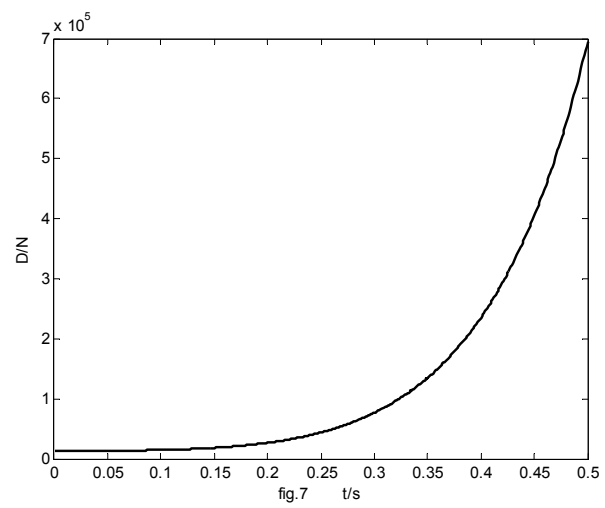

Fig 7 The curve of resistance

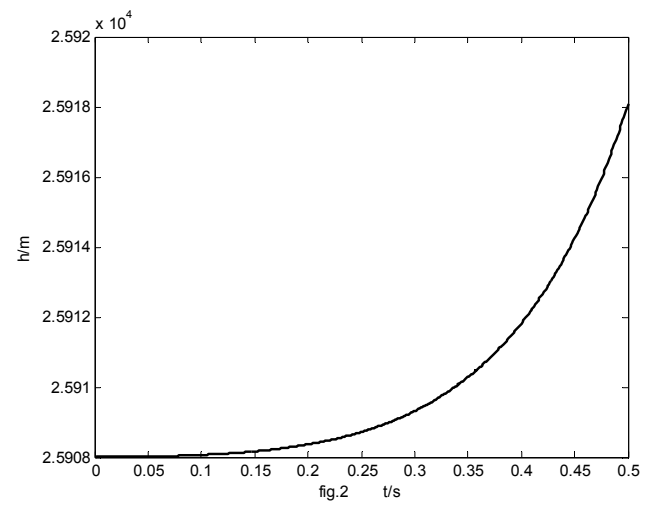

Fig 2 The curve of the height

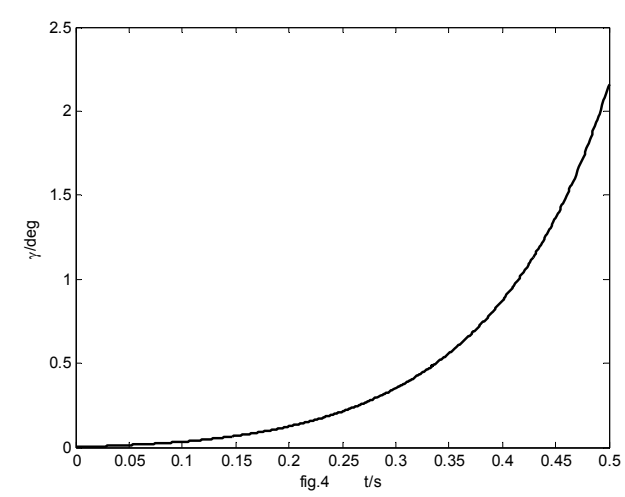

Fig 4 The curve of speed angle

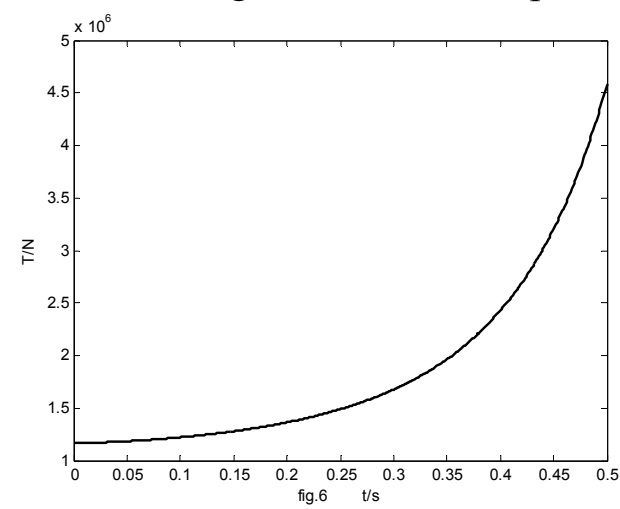

Fig 6 The curve of thrust

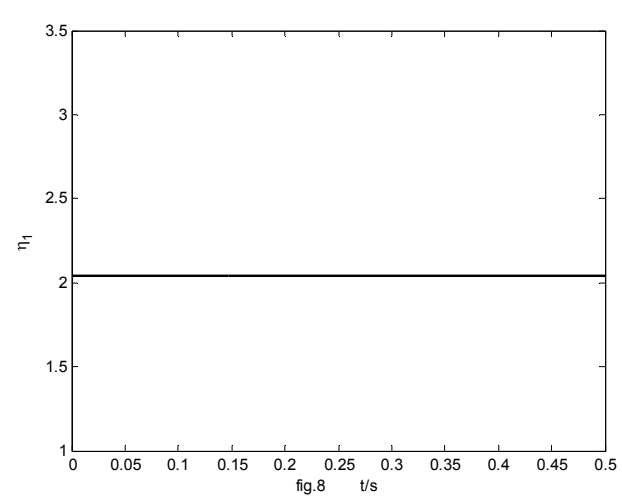

Fig 8 The state of first elastic shape 


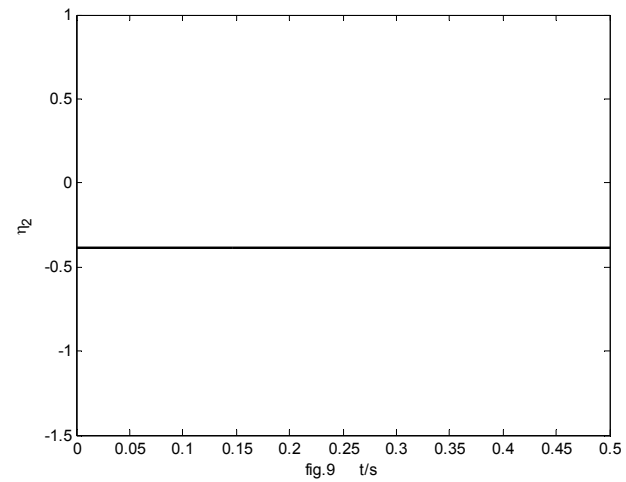

Fig 9 The state of second elastic shape

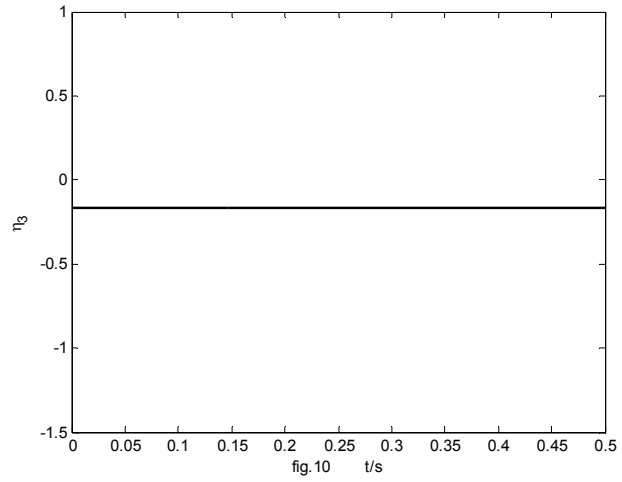

Fig 10 The state of third elastic shape

The simulation results show the dip angle of trajectory disperses the large degree, mainly as divergence of attack angle of projectile bodies. It's also may be the reason of increase sharply of the aircraft, lead to imbalance of moment. But the low speed missile can not appear this situation. The height and dip angle of trajectory all disperse slowly on the fixed attack angle.

\section{Conclusion}

The simulation shows that the spread of hypersonic missile attack angle is faster, It is not only caused by elastic form, the instability of the high-speed movement object itself also lead to the rapid spread of attack angle.Late study can be considered as a given attack angle,even given speed to study with elastic given, dynamic characteristic of hypersonic aircraft. But the speed change of hypersonic flight vehicle is not large from the perspective of the thrust and the speed change of engine, but the change of engine thrust is bigger, it may be affected by the coupling of the engine and attack angle, which resulted in increased thrust, torque imbalance and spread quickly. Hence the coupling of engine must be considered as another important factor besides hypersonic aircraft elastic deformation. The factor is stronger than the change of height and speed, and low speed missile has different nature.

\section{References}

[1] Holland S D, Woods W C, Engelund W C. Hyper-X research vehicle experimental aerodynamics test program overview[J]. Journal of Spacecraft and Rocket, 2001, 6(38):828-835.

[2] Chavez F R, Schmidt D K. Analytical aero-propulsive/aero-elastic hypersonic-vehicle model with dynamic analysis[J]. Journal of Guidance, Control, and Dynamics, 1994, 17(7): 1308-1319.

[3]Oppenheimer M W, Bolender M A. An overview on dynamic and controls modeling of hypersonic vehicles[C]//Proc. of American Control Conference. Orlando, AIAA press, 2009:25-38.

[4]Sigthorsson D O, Serrani A. Development of Linear Parameter-Varying Models of Hypersonic Air-Breathing Vehicles[C]//AIAA Guidance, Navigation, and Control Conference. AIAA Paper 10-13,2009

[5]Schmidt D K. Dynamics and control of hypersonic aeropropulsive/aeroelastic vehicles[A].In: AIAA Guidance,Navigation and Control Conference [C]. Washington, AIAA 92-4326.

[6] F. Chavez and D. Schmidt. Analytical Aeropropulsive/Aeroelastic Hypersonic Vehicle Model With Dynamic Analysis. Journal of Guidance,Control, and Dynamics. 1994,17(6):1308-1319.

[7]D.K.Schmidt. Ingegrated Control of Hypersonic Vehicles-A Necessity Not Just a Possibility. AIAA,1993:539-549.

[8]D.K.Schmidt. Optimum Mission Performance and Multivariable Flight Guidance for Airbreathing Launch Vehicles. Journal of Guidance, Control, and Dynamics, 
1997,20(6):1157-1164.

[9]Chavez F R and Schmidt D K.Flight dynamics and control of elastic hypersonic vehicles-Modeling uncertainties[A].In:AIAA Guidance, Navigation and Control Conference[C]. Washington,DC, AIAA 94-3629

[10]Chavez F R and Schmidt D K.Uncertainy modeling for multivariable-control robustness analysis of elastic high-speed vehicles $[\mathrm{J}]$. Journal of Guidance, Control,and Dynamics, 1999,22(1):87-95. 